\title{
Outpatient treatment in women with acute pyelo- nephritis after visiting emergency department
}

Hee Kyoung Choi ${ }^{1, *}$, Jin-Won Chung ${ }^{2}$, Won Sup Oh³ ${ }^{3}$ Jae-Bum Jun ${ }^{4}$, Yee Gyung Kwak ${ }^{5}$, Seong Yeon Park ${ }^{6}$, and Baek-Nam Kim ${ }^{7}$

\begin{abstract}
${ }^{2}$ Department of Internal Medicine, Wonju Severance Christian Hospital, Yonsei University Wonju College of Medicine, Wonju; ${ }^{2}$ Department of Internal Medicine, Chung-Ang University College of Medicine, Seoul; ${ }^{3}$ Department of Internal Medicine, Kangwon National University School of Medicine, Chuncheon; ${ }^{4}$ Department of Internal Medicine, Ulsan University Hospital, Ulsan; ${ }^{5}$ Department of Internal Medicine, Inje University Ilsan Paik Hospital, Goyang; ${ }^{6}$ Department of Internal Medicine, Dongguk University Ilsan Hospital, Goyang; ${ }^{7}$ Department of Internal Medicine, Inje University Sanggye Paik Hospital, Seoul, Korea
\end{abstract}

Received: December 21, 2015

Revised : January 13, 2016

Accepted: January 29, 2016

\section{Correspondence to}

Baek-Nam Kim, M.D.

Department of Internal Medicine, Inje University Sanggye Paik Hospital, 1342 Dongil-ro, Nowon-gu, Seoul 01757, Korea

Tel: $+82-2-950-8863$

Fax: +82-2-950-8883

E-mail: kimbn@paik.ac.kr

*Current affiliation: Healthcare Review and Assessment Committee, Health Insurance Review \& Assessment Service, Seoul, Korea
To the Editor, Acute pyelonephritis (APN) is a common infection of the kidney and renal pelvis in women but can progress to septic shock and multiple organ failure. In general, patients with mild APN who are able to take oral medication can be treated with oral antibiotics on an outpatient basis [1]. Recent American and European guidelines recommend hospitalization for patients with APN who are sufficiently ill (high fever, high white blood cell count, vomiting, dehydration, or evidence of sepsis). Traditionally, medical literature on the antimicrobial resistance, treatment, and outcomes of APN has focused on patients who are hospitalized with APN. Data on the epidemiology and clinical outcomes of APN patients discharged from the emergency department (ED) and subsequently treated on an outpatient basis are scarce. Therefore, we conducted this study to characterize the clinical characteristics and outcomes of APN patients discharged from the ED (outpatient treatment group) and to compare them with APN patients hospitalized from the ED (hospitalization group).

This observational cohort study was performed at six hospitals in South Korea. We prospectively identified women aged $>18$ years whose urine culture was performed in the ED and grew bacteria by screening the microbiology lab- oratory database daily at each hospital from June 29 to October 11, 2014. Polymicrobial cases were excluded and for each patient, only the first episode of APN [2] occurring during the study period was included. After reviewing the medical records, patients who had APN were enrolled in the study.

The subjects were followed up through each hospital's medical record system until hospital discharge or first outpatient follow-up visit. For the hospitalization group, treatment outcomes were assessed after 72 hours of treatment (early clinical success), and, if available, within 7 to 14 days after completion of antimicrobial therapy (final clinical cure). Clinical success was defined as resolution of fever and improvement of urinary tract symptoms or signs. For the outpatient treatment group, outcomes were evaluated at the time of first follow-up visit.

Of the 297 eligible women with APN who presented to the ED, two were excluded during the study period, because of immediate transfer to other hospitals. Of the remaining $295 \mathrm{pa}-$ tients who were included, 80 (27.1\%) were discharged from the ED and 215 (72.9\%) were hospitalized (Table 1). The admission rates ranged from $54.9 \%$ to 92.1\% in the six hospitals.

Compared with the hospitalization group, the outpatient treatment group 
Table 1. Clinical characteristics between outpatient treatment and hospitalization groups

\begin{tabular}{|c|c|c|c|}
\hline \multirow{2}{*}{ Characteristic } & \multicolumn{2}{|c|}{ Women with APN in emergency department } & \multirow{2}{*}{$p$ value $^{\mathrm{a}}$} \\
\hline & Outpatient treatment $(\mathrm{n}=8 \mathrm{o})$ & Hospitalization $(n=215)$ & \\
\hline Age, yr & $52(19-93)$ & $65(19-94)$ & $<0.001$ \\
\hline Presence of comorbidity & $36(45.0)$ & $155(72.1)$ & $<0.001$ \\
\hline Cardiovascular diseases & $22(27.5)$ & $89(41.4)$ & 0.029 \\
\hline Diabetes mellitus & $16(20.0)$ & $73(34.0)$ & 0.020 \\
\hline Neurologic diseases & $7(8.8)$ & $38(17.7)$ & 0.058 \\
\hline Presence of underlying condition & $11(13.8)$ & $63(29 \cdot 3)$ & 0.006 \\
\hline Admission within the preceding 90 days & $4(5.0)$ & $33(15 \cdot 3)$ & 0.017 \\
\hline Use of antibiotics within the preceding 90 days & $3(3.8)$ & $27(12.6)$ & 0.026 \\
\hline Bed-ridden state & $1(1.3)$ & $23(10.7)$ & 0.008 \\
\hline Complicated UTI & $6(7 \cdot 5)$ & $55(25.6)$ & 0.001 \\
\hline Healthcare-associated UTI & $15(18.8)$ & $53(24.7)$ & 0.285 \\
\hline Recurrent & $4(5.0)$ & $25(11.6)$ & 0.089 \\
\hline \multicolumn{4}{|l|}{ Clinical features at presentation } \\
\hline Duration of fever, day & $1(0-14)$ & $2(0-14)$ & 0.007 \\
\hline Chill & $50(62.5)$ & $158(73.5)$ & 0.066 \\
\hline Dysuria & $36(45.0)$ & $80(37.2)$ & 0.223 \\
\hline Frequency & $49(61.3)$ & $79(36.7)$ & $<0.001$ \\
\hline Urgency & $10(12.5)$ & $29(13.5)$ & 0.824 \\
\hline Suprapubic or perineal pain & $20(25.0)$ & $38(17.7)$ & 0.159 \\
\hline Flank pain & $27(33.8)$ & $89(41.4)$ & 0.232 \\
\hline Anorexia, nausea, or vomiting & $24(30.0)$ & $98(45.6)$ & 0.016 \\
\hline Tenderness on costovertebral angle & $66(82.5)$ & $156(72.6)$ & 0.079 \\
\hline Altered mental status & $4(5 \cdot 0)$ & $54(25.1)$ & $<0.001$ \\
\hline Systolic blood pressure $<90 \mathrm{mmHg}$ & $2(2.5)$ & $24(11.2)$ & 0.020 \\
\hline Heart rate $>90$ beats $/ \mathrm{min}$ & $32(40.0)$ & $121(56.3)$ & 0.013 \\
\hline Respiratory rate $>20$ breaths/min & $23(28.7)$ & $47(21.9)$ & 0.216 \\
\hline Temperature $>38^{\circ} \mathrm{C}$ or $<36^{\circ} \mathrm{C}$ & $48(60.0)$ & $157(73.0)$ & 0.031 \\
\hline \multicolumn{4}{|l|}{ Laboratory findings at presentation } \\
\hline WBC $>12,000$ or $<4,000 / \mu \mathrm{L}$ or band form $>10 \%$ & $26(32.5)$ & $116(54.0)$ & 0.001 \\
\hline Platelet $<150,000 / \mu \mathrm{L}$ & $9(11.4)$ & $52(24.2)$ & 0.016 \\
\hline BUN $>20 \mathrm{mg} / \mathrm{dL}$ or creatinine $>1.5 \mathrm{mg} / \mathrm{dL}$ & $18(22.5)$ & $89(41.4)$ & 0.004 \\
\hline SIRS criteria of $\geq 2^{b}$ & $42(52.5)$ & $154(71.6)$ & 0.002 \\
\hline Uropathogen & & & 0.078 \\
\hline Escherichia coli & $73(91.3)$ & $183(85.1)$ & \\
\hline Klebsiella pneumoniae & $1(1.3)$ & $12(5.6)$ & \\
\hline Proteus mirabilis & $2(2.4)$ & $7(3 \cdot 3)$ & \\
\hline Enterococci & 0 & $3(1.4)$ & \\
\hline Staphylococcus saprophyticus & $2(2.5)$ & 0 & \\
\hline Others $^{c}$ & $2(2.5)$ & $10(4.7)$ & \\
\hline ESBL production & $9 / 77(11.7)$ & $38 / 198(19.2)$ & 0.138 \\
\hline Concurrent bacteremia & $12 / 52(23.1)$ & $71 / 208(34.1)$ & 0.126 \\
\hline
\end{tabular}

Values are presented as median (range) or number (\%).

APN, acute pyelonephritis; UTI, urinary tract infection; WBC, white blood cell count; BUN, blood urea nitrogen; SIRS, systemic inflammatory response syndrome; ESBL, extended-spectrum $\beta$-lactamase.

${ }^{a}$ Mann-Whitney, chi-square, or Fisher exact tests as appropriate.

${ }^{\mathrm{b}}$ Two or more of the following criteria: (1) heart rate $>90$ beats $/ \mathrm{min},(2)$ respiratory rate $>20$ breaths $/ \mathrm{min}$, (3) temperature $>38^{\circ} \mathrm{C}$ or $<36^{\circ} \mathrm{C}$ or $(4)$ white blood cell $>12,000 / \mu \mathrm{L},<4,000 / \mu \mathrm{L}$, or band form $>10 \%$.

'Others included Acinetobacter baumannii, Citrobacter freundii, Enterobacter aerogenes, Gram-positive rod, Klebsiella oxytoca, Providencia rettgeri, Pseudomonas aeruginosa, Pseudomonas fluorescens, Serratia fonticola, Streptococcus agalactiae, and Streptococcus salivarius. 
was more likely to be younger (median age, 52 years vs. 65 years, $p<0.001$ ). These patients had less comorbidity, including cardiovascular diseases and diabetes mellitus. Previous hospitalization (5.0\% vs. 15.3\%, $p=0.017$ ) and antibiotic use (3.8\% vs. $12.6 \%, p=0.026$ ) before the first episode of APN during the study period were less commonly found in the outpatient treatment group. Further, the outpatient group more commonly presented with short median duration of fever ( 1 days vs. 2 days, $p=0.007$ ) and typical lower urinary tract symptoms (for example, frequency in $61.3 \%$ vs. $36.7 \%, p<0.001$ ). This group also less commonly had hypotension ( $2.5 \%$ vs. $11.2 \%, p=0.02)$ and altered mental status (5.0\% vs. $25.1 \%, p<0.001)$. Moreover, concurrent bacteremia was less common in the outpatient treatment group than in the hospitalization group (23.1\% vs. $34.1 \%, p=0.126$ ), although the difference was not statistically significant. Complicated episodes were less commonly found in the outpatient treatment group. However, the distribution of etiology was similar in both groups: enteric Gram-negative bacteria, particularly Escherichia coli, was the most frequent cause of APN. Extended-spectrum $\beta$-lactamase (ESBL)-producing gram-negative bacteria were less commonly the causative organisms in the outpatient treatment group than in the hospitalization group, although the difference was not statistically significant (11.7\% vs. 19.2\%, $p=0.138$ ).

Of the 80 patients in the outpatient treatment group, 61 were present for evaluation of clinical response at the first follow-up visit (Fig. 1). Among the 61 patients, 55 (90.2\%) showed clinical success at the first outpatient follow-up. In contrast, in the hospitalization group, early clinical success at 72 hours after empiric antibiotic therapy was observed in 183 patients (85.1\%). There was no significant difference in the clinical success between the two groups at the first assessment $(p=0.313)$, although the outcomes were not evaluated at the same follow-up time because of the natural limitation in the outpatient treatment group. Of 222 patients receiving appropriate empiric antibiotic therapy, 48 (92.3\%) of 52 discharged patients showed clinical success at the first outpatient visit and $150(88.2 \%)$ of 170 hospitalized patients showed early clinical success $(p=0.607)$. Of the 215 patients in the hospitalization group, final clinical cure was achieved in 163 (96.4\%) of 169 patients. Four patients (1.9\%) died in the hospital, two patients died due to APN 1 and 3 days after admission, one patient died of pneumonia 15 days after admission, and one patient died of heart failure 74 days after admission.

Nine (14.8\%) of the 61 discharged patients received inappropriate empiric antibiotic therapy on discharge from the ED (Table 2). The causative bacteria included E. coli in eight patients and Proteus mirabilis in one patient. Of those nine isolates, six were ESBL producers. The rate of clinical success at the first outpatient visit was lower in patients who received inappropriate empiric antibiotic therapy than in those who received appropriate empiric antibiotic therapy, but the difference was not statistically significant (77.8\% vs. $92.3 \%, p=0.212$ ). Two $(22.2 \%)$ of the nine patients receiving inappropriate empiric antibiotic therapy were admitted after the first outpatient visit as was the case with three $(5.8 \%)$ of the 52 patients receiving appropriate therapy, although these differences were not statistically significant $(p=0.154)$. The two cases that received inappropriate empiric antibiotic therapy and admitted after the first outpatient visit were caused by ESBL producers.

The results of this study presented an overview of the clinical characteristics, microbiology, and short-term treatment outcomes of adult women with APN who were

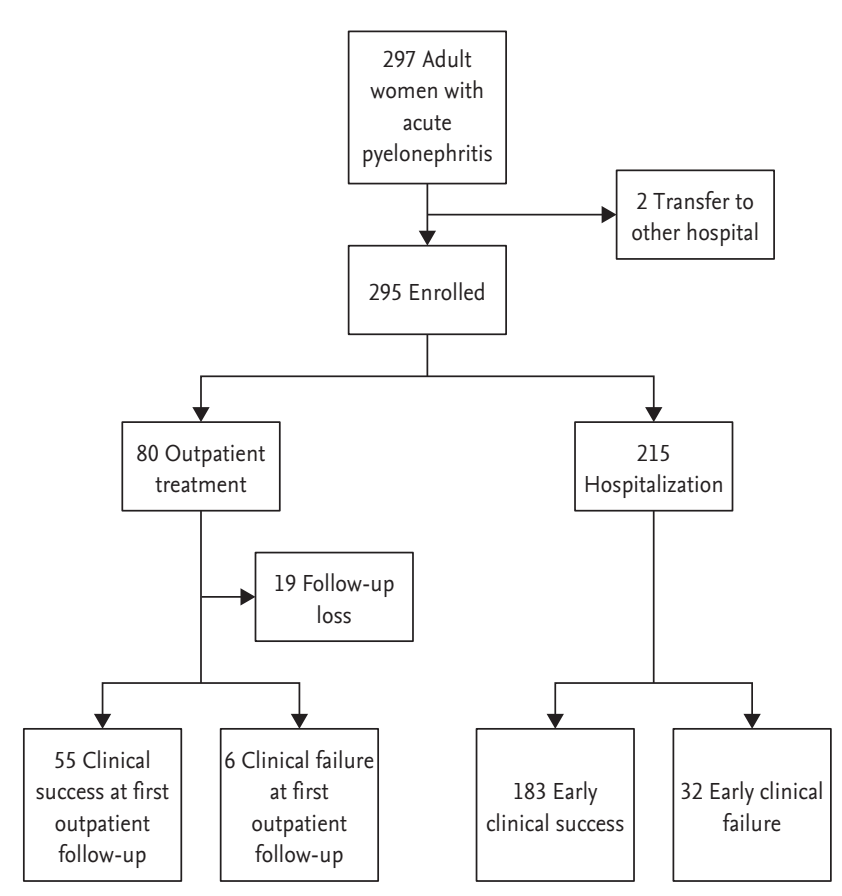

Figure 1. Flow of adult women with acute pyelonephritis presenting to emergency department. In the outpatient treatment group, the median follow-up interval from discharge to the first outpatient visit was 4 days (range, 1 to 8 ). 
Table 2. Outcomes according to appropriateness of empiric antibiotic therapy in the outpatient treatment group

\begin{tabular}{|c|c|c|c|}
\hline \multirow{2}{*}{ Outcome } & \multicolumn{2}{|c|}{ Empiric antibiotic therapy } & \multirow{2}{*}{$p$ value } \\
\hline & Appropriate $(\mathrm{n}=52)$ & Inappropriate $(\mathrm{n}=9)$ & \\
\hline Interval from ED discharge to first outpatient visit, day & $4(1-8)$ & $3(1-5)$ & $0.157^{\mathrm{a}}$ \\
\hline Clinical success at first outpatient visit & $48(92.3)$ & $7(77.8)$ & $0.212^{\mathrm{b}}$ \\
\hline Admission after first outpatient visit & $3(5.8)$ & $2(22.2)$ & $0.154^{\mathrm{b}}$ \\
\hline
\end{tabular}

Values are presented as median (range) or number (\%).

ED, emergency department.

${ }^{a}$ Mann-Whitney test.

${ }^{\mathrm{b}}$ Fisher exact test.

discharged from the ED. The overall admission rate in adult women with APN presenting to the ED was high (72.4\%) in this study; this rate is higher than that reported in a previous study in Korea [3]. Admission rates in patients with APN presenting to the ED vary widely depending on the country. In the Netherlands, $86 \%$ of adults with APN were hospitalized, while $26.9 \%$ of patients with APN in USA were hospitalized. To date, there has been no guideline or policy for admission of patients with APN in Korea, and the decision for admission is often subject to the physician's judgment. However, the admission rate for APN via the ED can be controlled or reduced: it decreased from $47.7 \%$ to $15.1 \%$ without complications after implementation of a standardized protocol for women with APN presenting to the ED [3].

In this study, as expected, the outpatient treatment group had milder APN than the hospitalization group. Significantly, this outpatient treatment group was less commonly exposed to known healthcare-associated risk factors for antimicrobial resistance, due to which ESBL producers were less commonly the causative organisms in this outpatient treatment group. These findings reflect slightly better outcomes in terms of clinical success and admission at the first outpatient visit in the outpatient treatment group, although the differences were not statistically significant.

In the outpatient treatment group, patients receiving inappropriate empiric antibiotic therapy had a lower clinical success rate at the first follow-up and a higher admission rate after the first outpatient visit, although the differences were not statistically significant. It is known that inappropriate empiric antibiotic therapy leads to worse early clinical response and longer hospital stay than appropriate therapy in bacteremic APN, although this therapy does not affect the mortality or clinical cure rate [4]. Interestingly, in this study, clinical failure and decision of admission at the time of first outpatient visit occurred only in patients whose condition was caused by ESBL producers. These findings are partly consistent with the findings of previous studies showing that inappropriate empiric antibiotic therapy for APN caused by ESBL-producing E. coli can delay recovery and result in longer hospitalization [5]. Recently, in Korea, third-generation cephalosporin-resistant uropathogens have been increasingly detected in women with community-associated APN, even without healthcare-associated factors [2].

Despite our important findings, this study has some limitations. First, we included only culture-proven cases, which might have led to an over- or under-estimation of the admission rate. Second, no information was available regarding the long-term results of the outpatient treatment group, and therefore, we were unable to directly compare the long-term outcomes (for example, final clinical success) between the outpatient treatment and hospitalization groups.

In conclusion, of adult women with APN presenting to the ED, the outpatient treatment group who were younger, had fewer comorbidities, and were less exposed to healthcare-associated factors were more likely to have mild infection. These patients can safely be treated on an outpatient basis without hospitalization; however, inappropriate empiric antibiotic therapy may delay recovery and hospitalization. Therefore, for optimal empiric antibiotic therapy, clinicians should always be vigilant to identify known risk factors for antimicrobial resistance before selecting empiric antibiotics for patients with APN presenting to the ED.

Keywords: Pyelonephritis; Emergency medical services; Ambulatory care 


\section{Conflict of interest}

No potential conflict of interest relevant to this article was reported.

\section{REFERENCES}

1. Warren JW, Abrutyn E, Hebel JR, Johnson JR, Schaeffer AJ, Stamm WE. Guidelines for antimicrobial treatment of uncomplicated acute bacterial cystitis and acute pyelonephritis in women: Infectious Diseases Society of America (IDSA). Clin Infect Dis 1999;29:745-758.

2. Park KH, Oh WS, Kim ES, et al. Factors associated with ciprofloxacin- and cefotaxime-resistant Escherichia coli in women with acute pyelonephritis in the emergency department. Int J Infect Dis 2014;23:8-13.

3. Kim K, Lee CC, Rhee JE, et al. The effects of an institutional care map on the admission rates and medical costs in women with acute pyelonephritis. Acad Emerg Med 2008;15:319-323.

4. Lee SS, Kim Y, Chung DR. Impact of discordant empirical therapy on outcome of community-acquired bacteremic acute pyelonephritis. J Infect 2011;62:159-164.

5. Lee S, Song do Y, Cho SH, Kwon KT. Impact of extended-spectrum beta-lactamase on acute pyelonephritis treated with empirical ceftriaxone. Microb Drug Resist 2014;20:39-44. 